\title{
STABLE QUOTIENTS OF PERIODIC MINIMAL SURFACES
}

\author{
MARTY ROSS AND CHAD SCHOEN
}

\begin{abstract}
Let $L \subset \mathbf{R}^{3}$ be a discrete lattice and suppose $x: M \rightarrow$ $\mathbf{R}^{3} / L$ is a complete and connected minimal immersion. Assuming that $x$ is stable and $M$ has finite genus we prove that if $\operatorname{rank} L=1$ or 2 then $x(M)$ is a quotient of the plane, the helicoid or a Scherk's surface. The proof combines minimal surface theory with techniques from algebraic geometry.
\end{abstract}

\section{The MAIN Theorem}

Suppose $L \subset \mathbf{R}^{3}$ is a discrete lattice and $x: M \rightarrow \mathbf{R}^{3} / L$ is a complete and connected minimal immersion. Such an $x$ lifts to (and may be obtained as the projection of or quotient of) a minimal immersion $\hat{x}: \widehat{M} \rightarrow \mathbf{R}^{3} ; \hat{x}$ need not be periodic but this will be the case if $x$ is proper ([MR, $\S 1]) . x$ is stable if the second variation of the area of $M$ (in the induced metric) is nonnegative for every compactly supported $C^{1}$ variation. By [FS], if $x$ is stable and orientable then $x(M)$ is totally geodesic (Th3, Cor4 and their proofs) and consequently lifts to a plane in $\mathbf{R}^{3}$. In this paper we investigate the case where $x$ is stable and nonorientable, and we give conclusions under the additional assumption that $M$ has finite genus.

In [LS] it is proved that a complete nonorientable minimal immersion in $\mathbf{R}^{3}$ with finite genus and infinite total Gaussian curvature is necessarily unstable: there is a compact set $K \subset \subset M$ such that $M-K$ is orientable, and thus Theorem 1 or Corollary 1 of $[F]$ can be applied. Since the results in $[F]$ apply to immersions into any flat 3-manifold (and much more generally), the arguments in [LS] apply mutatis mutandis. We conclude that if $x: M \rightarrow \mathbf{R}^{3} / L$

Second author partially supported by NSF grant DMS-90-14954 
is complete, nonorientable and has finite genus and infinite total curvature then $x$ is unstable.

If $M$ has finite total curvature we can begin by taking the orientable double cover $N$ of $M$ : if $\tilde{\pi}: N \rightarrow M$ is the natural projection then we obtain an immersion $\tilde{x}=x \circ \tilde{\pi}$. By Huber's Theorem, if we restrict to oriented isothermal parameters then $N=\bar{N}-\left\{p_{1}, \ldots, p_{k}\right\}$ is conformally a finitely punctured compact Riemann surface - in particular the assumption of finite total curvature automatically implies $M$ has finite genus. There is also an antipodal map $I: \bar{N} \rightarrow \bar{N}$, an anticonformal fixed-point free involution for which $\tilde{\pi} \circ I=\tilde{\pi}$. As well, because $N$ is orientable and $x(N) \subset \mathbf{R}^{3} / L$, there is a meromorphic Gauss map $G: N \rightarrow S^{2}$, and

$$
G \circ I=-G
$$

The existence of $G$ implies that a number of results for immersions into $\mathbf{R}^{3}$ may be established for immersions into $\mathbf{R}^{3} / L$ with only minor modifications in the proofs. Of specific interest to us are the following:

(a) ([O,Lemma 9.5], [Me1, §3.6]) $\quad G: \bar{N} \rightarrow S^{2}$ extends to a meromorphic function on all of $\bar{N}$;

(b) $([\mathrm{O}, \mathrm{Th} 9.2,9.3],[\mathrm{N}, \S 55])-2 \pi \operatorname{deg} G=\int_{M} K \leq 2 \pi \chi(M)$ where $K$ is the Gauss curvature of $M$ and $\chi(M)$ is the Euler characteristic of $M$;

(c) ([BCa]) If $\int_{M} K=-2 \pi$ then $x$ is stable;

(d) ([R1,Th 1]) Suppose there is a meromorphic function $H: \bar{N} \rightarrow S^{2}$ satisfying (1) and for which either

(i) $\operatorname{deg} H<\operatorname{deg} G$ or

(ii) $\operatorname{deg} H=\operatorname{deg} G$ and $H \neq T \circ G$ for any Möbius transformation $T: S^{2} \rightarrow S^{2}$.

Then $x$ is unstable.

(There is also a Weierstrass representation for nonorientable immersions into $\mathbf{R}^{3} / L$, which we apply in $\left.\S 3\right)$.

The inequality in (b) is known as Cohn-Vossen's inequality and holds for an arbitrary complete Riemannian 2-manifold of finite Euler characteristic. For a minimal immersion $x: M \rightarrow \mathbf{R}^{3}$ there is a precise version available [JM, Th 4]: 


$$
\int_{M} K=2 \pi(\chi(M)-n(M)) \quad x(M) \subset \mathbf{R}^{3},
$$

where $n(M)$ is the number of ends of $x(M)$ at $\infty$, counting multiplicity. Now, if $M$ is nonorientable then $n(M) \geq 3$ ([K,Cor1]). Therefore (2) and the equality in (b) imply that $\operatorname{deg} G \geq \gamma+3$ where $\gamma$ is the genus of $\bar{N}$. Thus, since $\bar{N}$ always admits meromorphic functions satisfying (1) of degree at most $\gamma+1$, it follows from (d)(i) that any nonorientable $x: M \rightarrow \mathbf{R}^{3}$ of finite total curvature is unstable.*

This result is the main conclusion (Th 3 ) of [R1], and it is the above argument which we attempt to adapt for immersions into $\mathbf{R}^{3} / L$. The one and only difficulty is that the degree of the Gauss map $G$ need not be so high. In general, if $\operatorname{rank} L=1$ or 2 one may have equality in (b) ([MR, Th 2], [Me1, Th $1.8]$ ), and of course if $\operatorname{rank} L=3$ and $x$ is a proper immersion then $M=\bar{M}$ is compact and equality in (b) follows from the Gauss-Bonnet Theorem. Thus the most we can obtain from (b) is $\operatorname{deg} G \geq \gamma-1$ if $\operatorname{rank} L=3$ and $\operatorname{deg} G \geq \gamma$ if $\operatorname{rank} L=1,2$. By [Ma, Lemma 1], $\operatorname{deg} G \equiv \gamma+1 \bmod 2$ and thus we can improve this to

$$
\begin{cases}\operatorname{deg} G \geq \gamma+1 & \operatorname{rank} L=1,2 \\ \operatorname{deg} G \geq \gamma-1 & \operatorname{rank} L=3\end{cases}
$$

We cannot apply (d)(i) (see, for example, [R1, Th 5, Cor 6]), but the following allows us to apply (d)(ii) in the first case above:

Theorem 1. Suppose $\bar{N}$ is a compact Riemann surface of genus $\gamma$ with antipodal map I. Then the space of meromorphic functions of degree $\gamma+1$ on $\bar{N}$ which satisfy (1) has real dimension $\gamma+3$.

The space of rotations of $S^{2}$ (= the space of Möbius transformations which preserve (1)) has real dimension 3. Therefore we can apply (d)(ii) when $\operatorname{rank} L=1$ or 2 and $\gamma \geq 1$. Analyzing the possibilities when $\gamma=0$, and

${ }^{*}$ In the context of minimal surfaces the estimate $\gamma+1$ on the degree is Lemma 2 of [R1]. More general results are given in [Ma], [G], and [GH]. The first author would like to thank the second author for bringing these references to his attention. 
combining with the work of [FS] and [LS], we obtain our conclusion regarding minimal surfaces:

Corollary 2. Suppose that $M$ is connected and has finite genus, and suppose that $x: M \rightarrow \mathbf{R}^{3} / L$ is a complete, stable minimal immersion. If rank $L=1$ or 2 then $x(M)$ is a quotient of the plane, the helicoid or a Scherk's surface.

Remarks.

(i) The maximal quotients of the helicoid and the Scherk's surfaces have total curvature $-2 \pi$ and thus are stable by (c). All other quotients are unstable by (b) and Theorem 1 .

(ii) If $\operatorname{rank} L=2$ and $L=\left\langle v_{1}, v_{2}\right\rangle$ is incommensurable (i.e. $L$ is not generated by two vectors of equal length) then $\mathbf{R}^{3} / L$ contains no Scherk's surface. In this case all proper stable immersions into $\mathbf{R}^{3} / L$ are derived from the plane. (Taking a quotient of the helicoid in $\mathbf{R}^{3} /\left\langle v_{1}\right\rangle$ and projecting into $\mathbf{R}^{3} / L$ gives rise to an improper stable immersion).

(iii) The classification problem when $\operatorname{rank} L=3$ is harder, as no simple analogue of Theorem 1 holds for functions of degree $\gamma-1$. Nonorientable quotients of Schwarz's $P$ and $D$ surfaces give stable genus 3 examples ([R2]), and these can be perturbed to give a 5-parameter family of stable embeddings ([Me2, Th 7.1]). We do not know if stable minimal surfaces of higher genus exist, but examples such as the Stessman surface ([FH,p581]) show that the methods of this paper will not apply in general. The Stessman surface is of genus 4 with a well-defined nonorientable quotient $x: \bar{M} \rightarrow \mathbf{R}^{3} / L$. The underlying Riemann surface is $w^{3}=z^{5}+z$ which admits, up to isometries of $S^{2}$, exactly one degree 3 meromorphic function satisfying (1) ([FK, III.8.7, VIII.3.1]). Thus $x$ cannot be shown to be unstable using (d)(ii). (That $x$ is in fact unstable can be shown by a direct test function argument).

(iv) It is an open question whether the assumption of finite genus is necessary, even for immersions into $\mathbf{R}^{3}$.

In the following section we prove Theorem 1 , and in $\S 3$ we classify the possibilities in the case $\gamma=0$. 


\section{Proof of Theorem 1}

The theorem will be proved by applying standard tools of algebraic geometry to real algebraic curves. Here the term real algebraic curve means one dimensional, non-singular, projective variety over the field of real numbers ([GH]), where by convention a variety must be geometrically irreducible [Ha, II.Ex 3.15]. Associated to each real algebraic curve is its field of rational functions. As is well known, the field of functions completely determines the curve, and there is in fact an equivalence of categories ([Si, Thm 2.4], [Ha, I.6]):

$$
\left\{\begin{array}{c}
\text { real algebraic } \\
\text { curves }
\end{array}\right\} \longleftrightarrow\left\{\begin{array}{c}
\text { finitely generated field extensions } \\
\mathbf{R} \subset F \text { satisfying } \\
1 . \text { trans. deg. } \mathbf{R}(F)=1 \text {, and } \\
2 . \mathbf{R} \text { is algebraically closed in } F .
\end{array}\right\} .
$$

Given a compact Riemann surface $\bar{N}$ of genus $\gamma$ with antipodal map $I$, write $\mathbf{F}_{N}$ for the field of global meromorphic functions, $F_{N}$ for the fixed field of the involution $h \rightarrow \overline{h \circ I}$ ( ${ }^{-}$denotes complex conjugation), and $X$ for the real algebraic curve corresponding to $F_{N}$ via (4). Similarly write $\mathbf{F}_{S}$ for the field of global meromorphic functions on $S^{2}, F_{S}$ for the fixed field of the involution $h(s) \rightarrow \overline{h(-s)}$, and $Q$ for the corresponding real algebraic curve. To give a holomorphic map $G: \bar{N} \rightarrow S^{2}$ is equivalent to giving a homomorphism of fields $\mathbf{F}_{S} \rightarrow \mathbf{F}_{N}$ which is the identity on the subfield of constant functions $\mathbf{C}$ ([FK, II.11.16, IV.11.17]). The existence of $G$ satisfying (1) is equivalent to the existence of a homomorphism $F_{S} \rightarrow F_{N}$ which is the identity on the field of constants R. By (4) this is equivalent to giving a morphism of real algebraic curves,

$$
g: X \rightarrow Q .
$$

Furthermore, giving such a map $g$ of degree $\gamma+1$ is equivalent to giving a subvariety (the graph of $g$ ) $\Gamma_{g} \subset X \times Q$, whose projection onto the first factor has degree 1 and whose projection onto the second factor has degree $\gamma+1$. The totality of all such (irreducible) subvarieties form an algebraic family

$$
p: \Gamma \longrightarrow \mathcal{H}
$$


where $\mathcal{H}$ is an open subset of the Hilbert scheme of $X \times Q\left([\mathrm{~S}, \S 7],[\mathrm{H}, \S 21]^{* *}\right)$. This family is non-empty ([Ma, Satz]). The tangent space to $\mathcal{H}$ at the point $[g] \in \mathcal{H}$ corresponding to the graph $\Gamma_{g}$ is canonically isomorphic to the space of global sections of the normal sheaf, $H^{0}\left(\Gamma_{g}, \mathcal{N}_{\Gamma_{g} / X \times Q}\right)$ ([S, 8.1]). Furthermore $\mathcal{H}$ will be non-singular at $[g]$ if $H^{1}\left(\Gamma_{g}, \mathcal{N}_{\Gamma_{g} / X \times Q}\right) \simeq 0([\mathrm{~S}, 8.6])$.

For any real algebraic curve $Y$ we write $\mathcal{O}_{Y}$ for the sheaf of regular functions, $\Omega_{Y / \mathrm{R}}$ for the sheaf of regular differential forms, and $\mathcal{T}_{Y}:=\underline{\operatorname{Hom}}_{\mathcal{O}_{Y}}\left(\Omega_{Y / \mathbf{R}}, \mathcal{O}_{Y}\right)$ for the tangent sheaf. Projecting $\Gamma_{g}$ onto $X$ gives isomorphisms

$$
H^{i}\left(\Gamma_{g}, \mathcal{N}_{\Gamma_{g} / X \times Q}\right) \simeq H^{i}\left(X, g^{*} \mathcal{T}_{Q}\right)
$$

Now we compute the degree of $\mathcal{T}_{Q}$ by noting that it is the same as the degree of the tangent sheaf of the corresponding complex curve, namely $\operatorname{deg} .\left(\mathcal{T}_{\mathrm{P}_{\mathrm{C}}^{1}}\right)=2$. Thus deg. $\left(g^{*} \mathcal{T}_{Q}\right)=2(\gamma+1)$ and deg. $\left(\Omega_{X / \mathbf{R}} \otimes\left(g^{*} \mathcal{T}_{Q}\right)^{-1}\right)=2 \gamma-2-2(\gamma+1)<0$. By Serre duality ([Se, II.8-10], [Fo, 17.9 $\left.]^{* *}\right)$

$$
H^{1}\left(X, g^{*} \mathcal{T}_{Q}\right) \simeq H^{0}\left(X, \Omega_{X / \mathrm{R}} \otimes\left(g^{*} \mathcal{T}_{Q}\right)^{\vee}\right)^{\vee} \simeq 0 .
$$

By Riemann-Roch ([I, 4.11;p. 187] or [Se, II.4], [Fo, 16.9]**)

$$
h^{0}\left(X, g^{*} \mathcal{T}_{Q}\right)=h^{0}\left(X, g^{*} \mathcal{T}_{Q}\right)-h^{1}\left(X, g^{*} \mathcal{T}_{Q}\right)=1-\gamma+\operatorname{deg} \cdot\left(g^{*} \mathcal{T}_{Q}\right)=\gamma+3 .
$$

Thus $\mathcal{H}$ is non-singular of dimension $\gamma+3$ ([S, 8.5]). The fibers of $p$ over the real points of the scheme $\mathcal{H}$ are precisely the graphs of the distinct morphisms $g: X \rightarrow Q$ of degree $\gamma+1$. These in turn are in bijective correspondence with holomorphic maps $G: \bar{N} \rightarrow S^{2}$ of degree $\gamma+1$ satisfying (1). Since the real points of $\mathcal{H}$ form a manifold of real dimension $\gamma+3$ the theorem follows.

\section{The Case of Genus 0}

Suppose $\bar{N}=\overline{\mathbf{C}}$ is of genus 0 . By (d)(i), we need only consider $\operatorname{deg} G=1$. Thus, by suitable choice of coordinates on $\bar{N}$, we can assume $g(z)=z$ where $g=\pi \circ G$ and $\pi: S^{2} \rightarrow \overline{\mathbf{C}}$ is stereographic projection. By (1), $I(z)=-1 / \bar{z}$. The Weierstrass representation for $x: N \rightarrow \mathbf{R}^{3} / L$ can now be written as

$$
x(p)=\operatorname{Re} \int_{p_{0}}^{p}\left(1-z^{2}, i\left(1+z^{2}\right), 2 z\right) \frac{d z}{P(z)},
$$

${ }^{* *}$ References marked ${ }^{* *}$ are introductory in nature and might be useful if additional background material is desired prior to consulting the actual reference needed. 
where $P(z)$ is a polynomial ([O, Lemma 9.5], [BCo, §2.1], [Me1, §3.6]). ((5) gives a multiple-valued immersion of $N$ into $\mathbf{R}^{3}$; the periods given by (5) are contained in $L$ because the integral represents a single-valued immersion of $N$ into $\mathbf{R}^{3} / L$. Similarly, because $x: N \rightarrow \mathbf{R}^{3} / L$ is invariant under $I$, integrals obtained by taking $p=I\left(p_{0}\right)$ are also contained in $\left.L\right)$. Since $x \circ I=x$, we have $([\mathrm{BCo}, \mathrm{p} 104])$

$$
z^{4} \overline{P(-1 / \bar{z})}=-P(z) .
$$

By (b), $\bar{M}$ has either one or two punctures, and thus $\bar{N}$ has either two or four punctures, occurring in antipodal pairs. This makes it easy to classify the possibilities for $P(z)$.

Suppose $\bar{M}$ has only one puncture. By rotating the immersion in $\mathbf{R}^{3}$ and making a corresponding change of coordinates on $\bar{N}$, we can assume that $N=$ $\mathbf{C}-\{0\}$. Then $P(z)$ can only have a zero at $z=0$, and thus $P(z)=c z^{k}$. (6) implies that $k=2$ and $c=\alpha i$ is purely imaginary, giving scalings of the helicoid.

Now suppose $\bar{M}$ has two punctures. We again rotate and change coordinates, after which we can assume the punctures of $\bar{N}$ occur at the points $e^{i \theta}, e^{-i \theta},-e^{i \theta},-e^{-i \theta}$, where $0<\theta \leq \pi / 4$. Then

$$
P(z)=c\left(z-e^{i \theta}\right)^{n_{1}}\left(z-e^{-i \theta}\right)^{n_{2}}\left(z+e^{i \theta}\right)^{n_{3}}\left(z+e^{-i \theta}\right)^{n_{4}}
$$

with each $n_{j} \geq 1$. (6) implies $\Sigma n_{j}=4$ and thus each $n_{j}=1$. It follows that $c$ is real and we obtain the Scherk surfaces.

\section{REFERENCES}

[BCa] Barbosa, J.L.M. and doCarmo, M.P., On the size of a stable minimal surface in $\mathbf{R}^{3}$, Amer. J. Math. 98 (1976) 515-528.

[BCo] Barbosa, J.L.M. and M.P. and Colares, A.G., Minimal surfaces in $\mathbf{R}^{3}$, Lecture Notes in Math. 1195 (1986), Springer-Verlag Berlin.

[F] Fischer-Colbrie, D., On complete minimal surfaces with finite Morse index in three manifolds, Invent. Math. 82 (1985), 121-132.

[FH] Fogden, A. and Hyde, S.T., Parametrization of triply periodic minimal surfaces. II. Regular class solutions, Acta Cryst. A48 (1992), 575-591.

[FK] Farkas, H. and Kra, I., Riemann Surfaces, Grad. Texts in Math. 71 SpringerVerlag, Berlin 1980.

[Fo] Forster, O., Lectures on Riemann Surfaces, Grad. Texts in Math., 81, SpringerVerlag, Berlin 1981. 
[FS] Fischer-Colbrie, D. and Schoen, R., The structure of complete stable minimal surfaces in 3-mainfolds of non-negative scalar curvature, Comm. in Pure and Appl. Math. 33 (1980), 199-211.

[G] Geyer, W.-D., Ein algebraischer Beweis des Satzes von Weichold über reelle algebraische Funktionkörper, in Algebraische Zahlentheorie 83-98 Math. Forsch., Oberwolfach 1964.

[GH] Gross, B.H. and Harris, J., Real algebraic curves, Ann. scient. Èc. Norm. Sup. 14 Ser. 4 (1981), 157-182.

[H] Harris, J., Algebraic Geometry, Grad. Texts in Math. 133, Springer-Verlag, New York 1992.

[Ha] Hartshorne, R., Algebraic Geometry, Springer-Verlag, New York 1977.

[I] Iitaka, S., Algebraic Geometry, Grad. Texts in Math. 76, Springer-Verlag, New York 1982.

[JM] Jorge, L.P.M. and Meeks III, W.H., The topology of complete minimal surfaces of finite total Gaussian curvature, Topology 22 (1983) 203-221.

[K] Kusner, R., Conformal geometry and complete minimal surfaces, Bull. Amer. Math. Soc. 17 (1987) 296-300.

[LS] Lima, I.C. and da Silveira, A.M., Stability of complete nonorientable minimal surfaces in $\mathbf{R}^{3}$, preprint.

[Ma] Martens, G., Minimale Blätterzahl bei Überlagerungen Kleinscher Flächen über der projectiven Ebene, Arch. Math. 30 (1978), 481-486.

[Me1] Meeks III,'W.H., The geometry, topology and existence of periodic minimal surfaces, Proc. Symp. Pure Math. 54 (1993) Part 1, A.M.S. 333-374.

[Me2] Meeks III, W.H., The theory of triply periodic minimal surfaces, Indiana Math. Jour. 39 (1990) 877-936.

[MR] Meeks III, W.H. and Rosenberg, H., The global theory of doubly periodic minimal surfaces, Invent. Math. 97 (1989), 351-379.

[N] Nitsche, J.C.C., Lectures on Minimal Surfaces, Vol. 1: Introduction, Fundamentals, Geometry and Basic Boundary Problems, Cambridge Univ. Press, 1989.

[O] Osserman, R., A Survey of Minimal Surfaces, 2nd Ed., Dover, New York, 1986.

[R1] Ross, M., Complete nonorientable minimal surfaces in $\mathbf{R}^{3}$, Comm. Math. Helv. 67 (1992), 64-76.

[R2] Ross, M., Schwarz' $P$ and D surfaces are stable, Diff. Geom. and its Appl. 2 (1992), 179-195.

[S] Sernesi, E,. Topics on Families of Projecitve Schemes, Queen's Papers in Pure and Applied Mathematics 731986.

[Se] Serre, J.-P., Algebraic Groups and Class Field, Grad. Texts in Math. 117, Springer-Verlag, New York 1988.

[Si] Silverman, J., The Arithmetic of Elliptic Curves, Grad. Texts in Math. 106, Springer-Verlag, New York 1986. 
LA Trobe Univeristy College of Northern Victoria, Australia

Current address: Melbourne University, Australia

E-mail address: marty@mundoe.maths.mu.oz.au

Duke UNIVERsity, U. S. A.

E-mail address: schoen@math.duke.edu

RECEIVED June 20, 1994. 